\title{
聚偏氟乙烯全反式分子链振动模式的研究 *
}

\author{
李吉超王春雷钟维烈 \\ (山东大学物理与微电子学院, 济南 250100)
}

\begin{abstract}
摘要 利用 $a b$ initio 分子轨道计算, 对 $\beta$ 相聚偏氟乙烯(PVDF)的全反式分子链进行了结构优化, 计算值和实验 值基本符合, 且发现 C $-\mathrm{C}-\mathrm{C}$ 键角有两种不同的值, 112. $8^{\circ}$ 和 113. $3^{\circ}$. 对该分子的频率进行了计算, 得到了红外 强度谱. 在 $400 \sim 4000 \mathrm{~cm}^{-1}$ 范围内, 对 $30 \mu \mathrm{m}$ 厚的聚偏氟乙烯取向膜和取向极化膜进行了红外光谱的测量, 红外吸收光谱和红外强度谱基本一致. 把所有振动模式分成六个系列: 系列 I(544 415 $\mathrm{cm}^{-1}$ ), 系列 II(913 $\left.792 \mathrm{~cm}^{-1}\right)$, 系列 III (1 $\left.353 \sim 998 \mathrm{~cm}^{-1}\right)$, 系列 IV (1 $\left.458 \sim 1361 \mathrm{~cm}^{-1}\right)$, 系列 V 和系列 VI. 其中只有系列 III 能改变分 子的电偶极矩, 该系列振动模式和 $\beta$ 相的 PVDF 的自发极化有着密切的关系.
\end{abstract}

关键词: 红外光谱, 聚偏氟乙烯, 振动模式, Ab initio 分子轨道计算 中图分类号: 0641

和其它无机铁电材料相比, 铁电聚合物聚偏氟 乙烯 (PVDF) 具有质轻、价廉及易于加工成大面积 薄膜的特点, 特别是其和三氟乙烯的无规共聚物 $\mathrm{P}(\mathrm{VDF}-\mathrm{TrFE})$ 经电子照射后的电致伸缩系数 $(Q)$ 由照射前的 $-2.1 \sim-2.5 \mathrm{~m}^{4} \cdot \mathrm{C}^{-2}$ 减少到 -13.5 $\mathrm{m}^{4} \cdot \mathrm{C}^{-2}$. 具有如此大的电致伸缩应变的材料在促 动器、传感器、换能器应用方面有明显的优势 ${ }^{[1-2]}$. 因而 PVDF 及其共聚物具有广泛的应用前景。近 年来众多学者对 PVDF 进行了详细研究. 实验观察 到 PVDF 有四种晶型, 分别为 $\alpha$ 相、 $\beta$ 相、 $\gamma$ 相和 $\delta$ 相 ${ }^{[3-4]} . \beta$ 相的 PVDF 极性最强, 是一种铁电体, 它和 三氟乙烯的共聚物是由链 $-\left(\mathrm{CH}_{2}-\mathrm{CF}_{2}\right)_{X}-\left(\mathrm{CF}_{2}-\right.$ $\mathrm{CHF})_{1-x}$-组成, 这条链在 $\mathrm{C}-\mathrm{C}$ 键的控制下, $\mathrm{F}$ 原 子对以 $0.26 \mathrm{~nm}$ 周期排列. 铁电相全反式结构的链 以准六角密排的形式平行排列, 属于正交晶系, 空间 点群为 $P m m 2^{[5]}$. 取向后的样品用粉末衍射的方法 得到它的精确的晶格常数 $c=(0.2560 \pm 0.0001)$ $n m^{[6]}$. 单体 $\left(\mathrm{CH}_{2} \mathrm{CF}_{2}\right)$ 的电偶极矩是 $\mathrm{CH}_{2}$ 和 $\mathrm{CF}_{2}$ 的 贡献之和. 实验对总体积中有一半为 $\beta$ 相的 PVDF 进行了电滞回线测量, 得到最大剩余极化为 $0.05 \sim$ $0.06 \mathrm{C} \cdot \mathrm{m}^{-2[4,6]}$. 七十年代 Kobayashi 等 ${ }^{[7]}$ 利用红 外光谱和 Raman 光谱对偏氟乙烯的振动模式进行 了研究, 结果发现 $\alpha$ 和 $\beta$ 相的 PVDF 空间点群分别 为 $C m 2 m$ 和 $P 2_{1} / c . \alpha 、 \beta$ 和 $\gamma$ 相的 PVDF 红外振
动模式的不同, 反映了三者分子链构象之间的差异. 红外和 Raman 光谱被广泛应用于研究不同成分的 无规共聚物 $\mathrm{P}(\mathrm{VDF}-\mathrm{TrFE})$ 的分子构象 ${ }^{[8-9]}$. 近年来 $\beta$ 相的红外振动模式又被广泛应用于判断被重离子 轰击过的 PVDF 中 $\mathrm{CC}$ 单键和双键的存在, 进而确 定重离子的轰击对 $\beta$ 相 PVDF 中晶体部分含量的

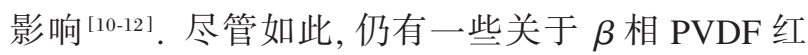
外振动模式的问题没有得到解决, 例如各种振动模 式的红外振动强度如何? 哪些模式可影响分子链的 电偶极矩? 这些问题的解决有利于更深人地了解 PVDF 的铁电性.

本文以解决以上问题为目的, 利用实验和 $a b$ initio 分子轨道计算相结合的方法对 $\beta$ 相的 PVDF 红外振动进行了详细研究.

\section{1 计算模型和方法}

考虑到 PVDF 是半晶态物质, 其晶态全反式的 分子链处在非晶包围之中, 所以选取一条由十个单 体 $\left(\mathrm{CH}_{2} \mathrm{CF}_{2}\right)$ 构成的分子链, $\mathrm{CH}_{3}\left(\mathrm{CF}_{2} \mathrm{CH}_{2}\right)_{9} \mathrm{CF}_{3}$ 作为 研究对象. 首先应用 Gaussian98W ${ }^{[13]}$ 程序包中限制 性的 Hartree-Fock (RHF) 方法对该分子链进行粗略 的估算, 发现计算值和实验结果基本符合. 在此基 础上再利用限制性的 B3LYP(RB3LYP), 即密度泛 函的方法对该体系进行详细的研究. 


\section{2 实 验}

PVDF 白色粉末是由上海有机氟研究所生产的 FR904, 其纯度大于 $97 \%$, 杂质为小于 $3 \%$ 左右的水. 将 FR904 溶于 $N, N$-二甲基甲酰胺溶剂中形成 15\% (质量分数)的溶液, 然后进行流延, 在真空条件 下, 温度保持 $90{ }^{\circ} \mathrm{C}, 0.5 \mathrm{~h}$ 后形成 $\alpha$ 相的 PVDF 薄 膜。这样保证了溶剂和水分能够完全蒸发, 因为 $N, N$ - 二甲基甲酰胺在 $5066.25 \mathrm{~Pa}$ 下的沸点为 76 ${ }^{\circ} \mathrm{C}$. 然后在 $150{ }^{\circ} \mathrm{C}$ 下将其拉伸为原来长度的 5 倍, 从而形成厚度为 $30 \mu \mathrm{m}$ 的 $\beta$ 相 PVDF 膜. 取一些 $\beta$ 相 PVDF 膜进行极化, 极化条件为, 温度 $90{ }^{\circ} \mathrm{C}$, 电 场 $60 \mathrm{kV} \cdot \mathrm{mm}^{-1}$, 极化时间 $50 \mathrm{~min}$. 在室温下, 利用 美国 Nicolet 公司制造的 FTIR、Magna-IR 型红外光 谱仪分别对极化和未极化的 $\beta$ 相 PVDF 膜在 400 $4000 \mathrm{~cm}^{-1}$ 频率范围内进行了红外光谱的测量.

\section{3 结果与讨论}

分别利用 RHF 和 RB3LYP 的方法 (基组选用 3-21G), 对分子链 $\mathrm{CH}_{3}\left(\mathrm{CF}_{2} \mathrm{CH}_{2}\right)_{9} \mathrm{CF}_{3}$ 进行结构优 化, 收玫精度采用系统默认值 $10^{-8}$ a. u. . 优化得 到具有稳定结构的分子体系的能量: $E($ RHF $)=$ -2842.420 a. u. ; $E(\mathrm{RB}+\mathrm{HF}-\mathrm{LYP})=-2855.922$ a. u. . 表 1 列出优化结果, 由表可以看出计算结果 和实验值 ${ }^{[6,14]}$ 基本符合, 且 RB3LYP 的结果比 RHF
更接近实验值. 计算中我们得到 $\mathrm{C}_{\mathrm{H}}-\mathrm{C}_{\mathrm{F}}-\mathrm{C}_{\mathrm{H}}$ 和 $\mathrm{C}_{\mathrm{F}}-\mathrm{C}_{\mathrm{H}}-\mathrm{C}_{\mathrm{F}}$ 键角的值不同, 且后者比前者略大, 而 实验 ${ }^{[14]}$ 没具体给出这两种键角。造成这两者不同 的可能原因是由于 $\mathrm{F}$ 原子的半径比 $\mathrm{H}$ 原子的半径 大, 且和 $\mathrm{C}$ 原子成键后的 $\mathrm{H}$ 和 $\mathrm{F}$ 原子所带总电荷分 别为 $0.24 e$ 和 $-0.28 e$ (RB3LYP 计算值). 所以相 邻 $\mathrm{F}$ 原子之间的库仑排斥比相邻 $\mathrm{H}$ 原子排斥强烈, 这样就造成 $C_{F}-C_{H}-C_{F}$ 的键角比 $C_{H}-C_{F}-C_{H}$ 的 键角大.

在此基础上, 对 PVDF 分子链进行了振动频率 的计算. 通过计算得到 180 个简正振动模式, 以及 它们的红外强度和振动的对称性。根据 Gaussian 分布, 取半高宽为 16 , 利用自编程序将振动频率和 红外强度转化成如图 1(a) 所示的红外强度曲线。 由图 1(a)可以看出, RHF 和 RB3LYP 的计算结果基 本一致, 但强度峰 III 上者有较大区别, 这将在本段 的下一部分进行详细讨论. 图 1(b) 是实验得到的 红外吸收曲线, 由此可以看出极化对 $\beta$ 相 PVDF 的 红外吸收光谱几乎没有影响. 比较图 1(a) 和 1 (b), 可以看出计算值和实验值基本一致. 图 1(a) 中峰 I的频率范围是 $512 \sim 399 \mathrm{~cm}^{-1}$, 和实验观察到 的吸收峰 I(图 1(b)) 的频率范围 $544 \sim 415 \mathrm{~cm}^{-1}$ 基 本一致. 我们称这一系列的振动模式为系列 I. 图 1 (a)中的峰 III对应着系列 II振动模式, 计算得到的频

表 1 PVDF 分子结构的计算值和实验值

Table 1 The calculated and observed results of geometric structure of PVDF molecule

\begin{tabular}{|c|c|c|c|}
\hline & Calculated results of RHF & Calculated results of RB3LYP & Experiment \\
\hline & & Bond length $(\mathrm{nm})$ & \\
\hline $\mathrm{C}-\mathrm{C}$ & 0.152 & 0.153 & $0.154^{[14]}$ \\
\hline $\mathrm{C}-\mathrm{H}$ & 0.108 & 0.109 & $0.109^{[14]}$ \\
\hline \multirow[t]{2}{*}{$\mathrm{C}-\mathrm{F}$} & 0.137 & 0.139 & $0.134^{[14]}$ \\
\hline & & Bond angle $\left({ }^{\circ}\right)$ & \\
\hline $\mathrm{H}-\mathrm{C}-\mathrm{H}$ & 109.5 & 109.6 & $112^{[14]}$ \\
\hline $\mathrm{F}-\mathrm{C}-\mathrm{F}$ & 107.7 & 108.3 & $108^{[14]}$ \\
\hline $\mathrm{C}_{\mathrm{H}}-\mathrm{C}_{\mathrm{F}}-\mathrm{C}_{\mathrm{F}}$ & 112.1 & 112.8 & $112.7^{[14] *}$ \\
\hline $\mathrm{C}_{\mathrm{F}}-\mathrm{C}_{\mathrm{H}}-\mathrm{C}_{\mathrm{F}}$ & 113.6 & 113.3 & \\
\hline \multirow[t]{5}{*}{$C-C-C-C$} & 159.5 & 164.2 & 171. $6^{[14]}$ \\
\hline & & $d_{\mathrm{F}} / \mathrm{nm}$ & \\
\hline & 0.273 & 0.268 & $0.26^{[14]}$ \\
\hline & & $d_{\mathrm{m}} / \mathrm{nm}$ & \\
\hline & 0.2562 & 0.2567 & $0.256 \pm 0.0001^{[6]}$ \\
\hline
\end{tabular}

$\mathrm{C}_{\mathrm{H}}$ and $\mathrm{C}_{\mathrm{F}}$ denote the $\mathrm{C}$ atom bonding with $\mathrm{H}$ and $\mathrm{F}$ atoms, respectively. ${ }^{*}$ Only bond angle of $\mathrm{C}-\mathrm{C}-\mathrm{C}$ was observed in experiment, the two different kinds of C-C-C were not observed. $d_{\mathrm{F}}$ denotes the distance of neighboring $\mathrm{F}$ atom pairs; $d_{\mathrm{m}}$ denotes the distance of neighboring monomers. 


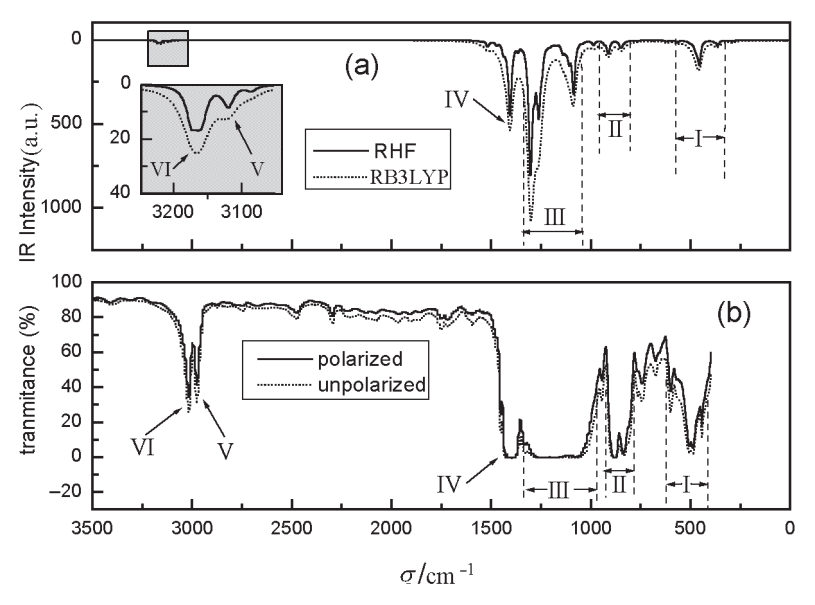

图 1 计算得到的红外强度曲线 (a)及 $\boldsymbol{\beta}$ 相 PVDF 薄膜的 红外光谱 (b)

Fig. 1 The calculated IR intensity spectra (a) and the IR spectra of $\beta$-phase PVDF (b)

率范围是 $920 \sim 801 \mathrm{~cm}^{-1}$. 实验上我们观察到吸收 峰 II (图 1(b)) 的频率范围是 $913 \sim 792 \mathrm{~cm}^{-1}$, 两者 符合得较好. 比较图 1(a) 和 1(b) 的峰 III 可以看出, 图 1(a)中峰 III 有较大的红外强度, 同时吸收峰 III (图 1(b))表现出强烈的吸收, 这表明计算和实验是 一致的. 计算得到的系列 III 振动模式的强度曲线形 状和实验观察到的吸收峰 III 的形状有一定差别, RHF 的计算结果显示在 $1349 \sim 1027 \mathrm{~cm}^{-1}$ 频率范 围内有三个强度峰。而 RB3LYP 的计算结果是在 相同的频率范围内有两个强度峰, 且这两个峰的宽 度比由 RHF 得到的峰宽度要大. 和 RHF 的结果相 比较, 由 RB3LYP 得到的两个峰相距更近, 有合并
趋势。实验得到的吸收谱(图 1(b)) 在 $1353 \sim 998$ $\mathrm{cm}^{-1}$ 频率范围内只有一个较宽的吸收峰, 造成这种 差别的原因可能是由于该系列中的两个强度峰 (RB3LYP 的结果) 频率比较靠近, 所以二者合成一 个比较宽的吸收峰。我们是基于以下原因而这样 认为的: 首先, 计算得到的频率范围 (1 349 1 027 $\mathrm{cm}^{-1}$ ) 和实验观察到的吸收峰 III 的频率范围(1353 $998 \mathrm{~cm}^{-1}$ ) 是一致的; 其次, 利用 RB3LYP 方法计算 得到的系列 III 强度曲线的形状和标准谱 ${ }^{[15]}$ 的形状 吻合得较好. 图 1(a)中, 系列 IV 振动模式的频率范 围是 $1459 \sim 1381 \mathrm{~cm}^{-1}$, 这和实验观察到的吸收峰 IV 的频率范围 1458 1361 cm $\mathrm{cm}^{-1}$ 基本一致. 从图 1 (a)中可以看到系列 $V$ 和 VI的振动模式的红外强度 比较小, 这对应着图 I(b) 中吸收峰 $\mathrm{V}$ 和 VI的较弱的 吸收。

系列 I的振动模式是氢原子和与之相连的碳原 子在垂直于链轴的方向来回摇摆, 同时伴随着碳氟 键的轻微摆动, 如图 2(a)所示. 通过查看计算结果 中是否存在分子电偶极矩对该系列振动模式对应的 简正坐标的微分的单位矢量, 我们发现系列 I的简 正振动模式不能改变分子链的电偶极矩大小. 系列 II振动模式中的振动是氢原子和与之相连碳原子在 垂直于链的方向上下振动, 并且相邻单体的碳、氢原 子的振动方向总是相反的, 即相位差为 $180^{\circ}$, 如图 2 (b)所示. 计算结果中无分子电偶极矩对该系列振 动模式的简正坐标的微分的单位矢量, 这表明系列 II的简正振动模式对 PVDF 分子链的电偶极矩的改 变没有贡献. 系列 III 振动模式是, 和氟原子相连的

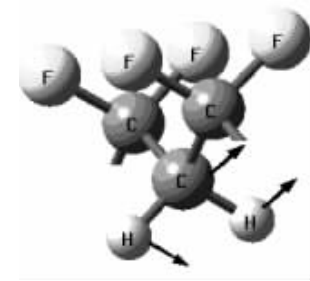

(a)

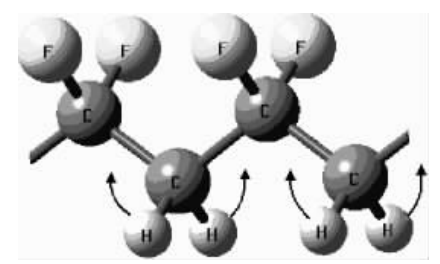

(d)

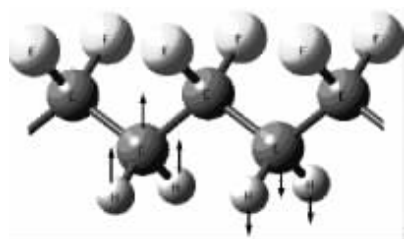

(b)

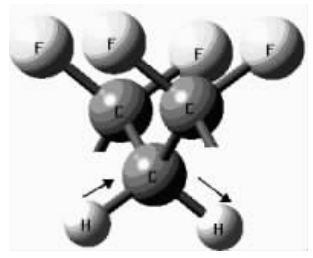

(e)

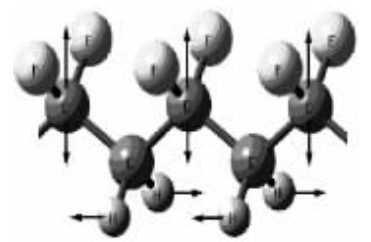

(c)

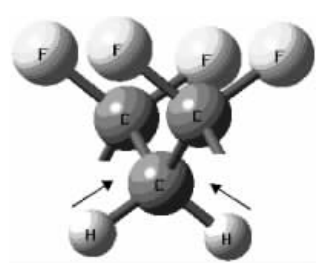

(f)

图 2 PVDF 各种振动模式的图解

Fig. 2 Schematic of all kinds of vibrational modes

$\mathrm{a} \sim \mathrm{f}$ correspond to series $\mathrm{I} \sim \mathrm{VI}$ vibrational modes 


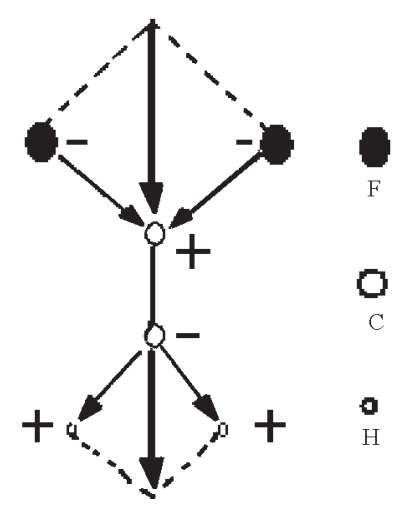

图 3 单体 $\mathrm{CF}_{2}=\mathrm{CH}_{2}$ 电偶极矩的图解

Fig. 3 Schematic of the electric dipole of monomer $\mathrm{CF}_{2}=\mathrm{CH}_{2}$

碳原子 $\left(\mathrm{C}_{\mathrm{F}}\right)$ 沿垂直链的方向上下振动, 同时伴随着 氢原子的摇摆运动, 如图 2(c) 所示. 前一种振动较 强烈, 是该模式中的主要振动方式, 后一种振动较微 弱,对该模式的影响不大．计算结果表明,系列 III 振动模式造成 PVDF 分子链的电偶极矩的改变. 由计算结果可得到 PVDF 分子中各原子所带电荷的 性质: 氢原子以及和氟原子相连的碳原子 $\left(\mathrm{C}_{\mathrm{F}}\right)$ 带正 电荷, 氟原子以及和氢原子相连的碳原子 $\left(\mathrm{C}_{\mathrm{H}}\right)$ 带负 电荷。一个单体的电偶极矩是两个 $\mathrm{CH}$ 键和两个 CF 键所形成的电偶极矩的矢量和, 如图 3 所示. 由 图 3 可以看出单体的电偶极矩垂直于分子链的方向 而指向氢原子所在的一边. 单体的电偶极矩平行排 列构成了整个分子链的电偶极矩. 计算结果中给出 了不同方向的电偶极矩, 列于表 2 . 由表 2 可以看出 沿 $y$ 轴方向分子的电偶极矩绝对值最大, 这和以上 的分析结果是一致的. 从微观角度讲, 作为铁电体 的 PVDF 的自发极化是其分子的电偶极矩按一定对 称性堆积排列的结果. 所以分子链的电偶极矩垂直 于链的方向和实验观察到的自发极化的方向沿晶格 的 $b$ 轴方向是一致的 ${ }^{[3]}$. 在系列 III 振动模式中, $\mathrm{C}_{\mathrm{F}}$ 原子向上运动时, $\mathrm{CF}$ 键的键长变小, 同时 $\mathrm{F}-\mathrm{C}-\mathrm{F}$ 键角就会变大. 这样两个 $\mathrm{CF}$ 键所形成的电偶极矩

\section{表 2 计算得到的分子的不同方向的电偶极矩 $\left(10^{-30} \mathrm{C} \cdot \mathrm{m}\right)$}

Table 2 The calculated PVDF molecular dipole moment in different directions

\begin{tabular}{cccc}
\hline $\begin{array}{c}\text { Dipole moment } \\
\text { along } x \text {-axis }\end{array}$ & $\begin{array}{c}\text { Dipole moment } \\
\text { along } y \text {-axis }\end{array}$ & $\begin{array}{c}\text { Dipole moment } \\
\text { along } z \text {-axis }\end{array}$ & $\begin{array}{c}\text { Total dipole } \\
\text { moment }\end{array}$ \\
\hline-7.1012 & -27.2554 & 4.2897 & 28.4902 \\
\hline$x$-axis is along the direction of molecular chain, $y$-axis is perpendicular
\end{tabular}
to the direction of molecular chain, and points to the side of $\mathrm{F}$ atoms.
都会变小, 又由于 $\mathrm{F}-\mathrm{C}-\mathrm{F}$ 键角的变大, 两者的矢 量和会变得更小, 结果造成单体的电偶极矩变小. 相反, 当 $\mathrm{C}_{\mathrm{F}}$ 原子向下运动时, $\mathrm{CF}$ 键的键长变长, 同 时 $\mathrm{F}-\mathrm{C}-\mathrm{F}$ 键角变小, 这会造成和 $\mathrm{C}_{\mathrm{F}}$ 原子向上运 动相反的结果, 即单体的电偶极矩变大. 所以该系 列振动模式影响了 PVDF 单体的电偶极矩的大小.

PVDF 的自发极化是其分子的电偶极矩按一定规律 排列的结果, 所以系列 III 振动模式直接影响聚合物 的自发极化强度的大小. 因此我们认为该系列的振 动模式和聚合物的铁电性有着密切的联系. 系列 IV 振动模式是碳氢键做弯曲运动, 同时伴随着碳碳键 轻微的弯曲运动, 如图 2(d) 所示. 计算结果表明, 该系列振动模式不能改变 PVDF 分子的电偶极矩. 碳氢键在系列 $\mathrm{V}$ 中做反对称伸缩, 即氢原子沿碳氢 键的方向作反对称运动, 其余原子静止不动, 如图 2 (e) 所示. 然而碳氢键在系列 V振动模式中的伸缩 运动是对称的, 即氢原子沿碳氢键的方向对称振动, 如图 2(f)所示. 计算结果表明,系列 $\mathrm{V}$ 和 $\mathrm{VI}$ 简正振动 模式不能改变 PVDF 分子的电偶极矩. 由以上分析 可知, 在 $400 \sim 4000 \mathrm{~cm}^{-1}$ 频率范围, 只有系列 III 振动 模式对 PVDF 分子的电偶极矩变化有贡献, 该系列振 动模式和 PVDF 的自发极化有着密切的关系.

\section{4 结 论}

利用 $a b$ initio 分子轨道计算法对 PVDF 全反式 分子链进行了结构优化, 计算值和实验值基本符合, 同时发现了两种不同的 $\mathrm{C}-\mathrm{C}-\mathrm{C}$ 键角。通过对 PVDF 分子链的频率计算, 我们得到了和红外吸收 谱符合较好的红外强度谱. 在 $400 \sim 4000 \mathrm{~cm}^{-1}$ 频 率范围内, 所有的振动模式分成六个系列. 其中只 有系列 III (1 $\left.353 \sim 998 \mathrm{~cm}^{-1}\right)$ 能够改变 PVDF 分子 的电偶极矩, 这和聚合物的自发极化有着密切的联 系. 铁电聚合 PVDF 的简正振动随温度的变化有待 于进一步深人研究, 以便能够更好地解释其自发极 化强度随温度变化的微观机制, 进而解释其铁电 顺电相变行为 .

\section{Referenes}

1 Zhang, Q. M. ; Bharti, V. ; Zhao X. Science, 1998, 280: 2101

2 Cheng, Z. Y. ; Bharti, V. ; Mai, T. ; Xu, T. B. IEEE Trans. Ultras., Ferroelect., Freq. Contr., 2000, 47: 1296

3 Kepler, R. G. ; Anderson, R. A. Adv. in Phys., 1992, 41: 1

4. Furukawa, T. Ferroelectrics, 1990, 104: 229

5 Legrand, J. F. Ferroelectrics, 1989, 91: 303 
6 Bellet-Amalric, E. ; Flegrand, J. Eur. Phys., 1998, B3: 225

7 Kobayashi, M. ; Tashiro, K. ; Tadokoro, H. Macromol., 1975, 8: 161

8 Tashiro, K. ; Takano, K. ; Kobayashi, M. ; Chatani, Y. ; Tadokoro, H. Ferroelectrics, 1984, 57: 297

9 Tashiro, K. ; Kobayashi, M. Phase Transition, 1989, 18: 213

10 Balanzat, E. ; Bouffard, S. ; Moel, A. L. ; Betz, N. Nucl. Instrum. Methods Phys. Res., 1994, B91: 140

11 Bouedec, A. L. ; Betz, N. ; Esnouf, S. ; Moel, A. L.
Nucl. Instrum. Methods Phys. Res., 1999, B151: 89

12 Lotham, S. ; Fink, D. ; Paul, S., Bose, S. K. Appl. Phys. Lett., 2001, 78: 4136

13 Gaussian98W. Pittsburgh, PA: Gaussian, 1998

14 Hasagawa, R. ; Takashi, Y. ; Chatani, Y. ; Tadokoro, H. J. Polym., 1972, 3: 6000

15 Hummel, D. D. Atlas of polymer and plastic analysi. Vol. 1. Weinheim: VCH Verlagsgesellschaft GmbH, 1985: 36

\title{
Vibrational Modes Analysis of Poly (vinylidene fluoride) All-trans Molecular Chain*
}

\author{
Li Ji-Chao Wang Chun-Lei Zhong Wei-Lie \\ ( School of Physics and Microelectronics, Shandong University, Jinan 250100)
}

\begin{abstract}
Ab initio molecular orbital calculation was employed to optimized the geometry structure of poly (vinylidine fluoride) (PVDF) all-trans molecule. The calculated result is consistent with that of experiment. In our calculation, two different values of bond angle $\mathrm{C}-\mathrm{C}-\mathrm{C}$ were found, i. e. $112.8^{\circ}$ and $113.3^{\circ}$. The infrared intensity spectrum was obtained from frequencies calculation of the all-trans molecule. In the range of $400 \sim 4000 \mathrm{~cm}^{-1}$, the IR spectra of polarized and unpolarized $\beta$-phase PVDF film (30 $\mu \mathrm{m}$ thick) have been measured. The calculated IR intensity spectrum is in good agreement with observed IR spectrum. The vibrational modes in the range of $400 \sim 4000 \mathrm{~cm}^{-1}$ were divided into six series, series I $\left(544 \sim 415 \mathrm{~cm}^{-1}\right)$, series II $\left(913 \sim 792 \mathrm{~cm}^{-1}\right)$, series III ( $1353 \sim 998 \mathrm{~cm}^{-1}$ ), series IV (1 $458 \sim 1361 \mathrm{~cm}^{-1}$ ), series V and VI. Only series III of all the vibrational modes causes the change of the dipole moment of PVDF molecule, and is related to the spontaneous polarization.
\end{abstract}

Keywords: IR spectrum, PVDF, Vibrational modes, Ab initio molecular orbital calculation 